\title{
Software to calculate calorie content of food and its application in medical practice
}

\author{
Dr. B.R. Booso \\ Postgraduate Institute of Medicine, University of Colombo, Sri Lanka. \\ Dr. E.S.S. Rodrigo \\ Postgraduate Institute of Medicine, University of Colombo, Sri Lanka. \\ Dr. B.K.N. Fernando \\ Postgraduate Institute of Medicine, University of Colombo, Sri Lanka. \\ Contact e-mail address: semuthurodrigo@gmail.com \\ eHealth Sri Lanka 2010,1(suppl.1):S34 \\ DOI: http://dx.doi.org/10.4038/sljbmi.v1i0.3593
}

Only the Abstract is available

\begin{abstract}
The government of Sri Lanka acknowledges that the prevention and control of chronic NCDs is a priority issue in the national health agenda in the National Health Master Plan 2007-2016. Diet and nutrition are closely related to general population health and the burden of non-communicable diseases. To maintain good health, nutritionally balanced diet with appropriate amount of energy is required. To measure dietary energy, quantitative food intake data are generally converted into nutrient terms and compared with a suitable standard, usually a recommended dietary or minimal dietary requirement. Presently, there is no standard method to calculate dietary nutrient amount in Sri Lankan food and most of the time it is done manually.

Therefore, the aim of introducing this simple medically oriented software is to enable computerised assessment of calorie content in Sri Lankan food. The database of this software consists of calorie content and other data of food that is nutritionally important and consumed in Sri Lanka. It can be updated with latest data taken from the web or any other latest research to get the maximum output.

Total amount of calorie intake for a day can be assessed when the types of food in a meal and their relevant quantities are entered to this software. It makes easy for the medical practitioner to find out whether the patient needs dietary modification in order to achieve a balanced diet with adequate calories In addition, this software is incorporated to produce a dietary plan for each type of patient according to the individual preference. So the patients can create their own dietary plan with or without the assistance of a dietician. In fact, this software can be used by doctors, dieticians as well as patients themselves.
\end{abstract}

Keywords - calorie content of food, medical practice 